\title{
Age-Related Serum Total Immunoglobulin E Levels in Suspected Allergic People in Bangladesh
}

\author{
Sabrina Afrin1, Mushtaque Ahmed1, Nabeela Mahboob', Hasina Iqbal', Md. Kamrul Hasan², \\ Md. A. K. Shameem ${ }^{3}$, Kamrun Nahar Islam ${ }^{4}$, Sifat Uz Zaman ${ }^{5 *}$ \\ ${ }^{1}$ Department of Microbiology, Popular Medical College, Dhaka, Bangladesh \\ ${ }^{2}$ Department of Management, School of Business, North South University, Dhaka, Bangladesh \\ ${ }^{3}$ Department of Laboratory Medicine, Biomed Laboratory, Dhaka, Bangladesh \\ ${ }^{4}$ Department of Microbiology, University of Chittagong, Chittagong, Bangladesh \\ ${ }^{5}$ Infection Prevention and Control Division, Medlife Healthcare Limited, Dhaka, Bangladesh \\ Email: *sifat.zaman9@gmail.com
}

How to cite this paper: Afrin, S., Ahmed, M., Mahboob, N., Iqbal, H., Hasan, Md.K., Shameem, Md.A.K., Islam, K.N. and Zaman, S.U. (2022) Age-Related Serum Total Immunoglobulin E Levels in Suspected Allergic People in Bangladesh. Journal of Biosciences and Medicines, 10, 13-20.

https://doi.org/10.4236/jbm.2022.103003

Received: January 26, 2022

Accepted: February 28, 2022

Published: March 3, 2022

Copyright $\odot 2022$ by author(s) and Scientific Research Publishing Inc. This work is licensed under the Creative Commons Attribution International License (CC BY 4.0).

http://creativecommons.org/licenses/by/4.0/

\begin{abstract}
Allergy is an adverse immunologic response of increasing prevalence and severity in which immunoglobulin E plays a pivotal role. Total IgE (tIgE) levels show wide variations depending on various factors, such as age, sex, ethnicity, and geographic area. This study set out to quantitatively determine the serum tIgE levels in Bangladeshi population who were clinically suspected cases of allergic diseases and to find out the association of tIgE level with their age and gender. This cross-sectional descriptive exploratory study was conducted at Biomed Diagnostics Laboratory, Panthapath, Dhaka, from March 2021-September 2021. A total of 402 blood samples were collected from clinically suspected cases of allergic diseases. Quantitative detection of tIgE in serum was done by Immunoblot assay manufactured by Proteome Tech Inc. (PROTIA ${ }^{\mathrm{TM}}$ Allergy-Q64S panel). Among 402 subjects, a wide variation of tIgE levels ranging from 5 to 2000 $\mathrm{IU} / \mathrm{ml}$ was found but majority of the study population (30.35\%) showed IIgE levels within the range of $201-350 \mathrm{IU} / \mathrm{ml}$. The study population aging $1-10$ years showed highest frequency (23.63\%) in tIgE concentrations and the mean $\operatorname{tgE}$ level exhibited a declining tendency with an increase in age. Depending on gender there was no difference in tIgE levels. Determination of tIgE level is imperative for screening allergic diseases. The present study enlightened the distribution of tIgE levels and suggested that among Bangladeshi subjects irrespective of gender there is an inverse relationship between $\operatorname{tgE}$ level and age.
\end{abstract}

\section{Keywords}

Allergy, Serum Total IgE, Bangladesh, Age, Gender 


\section{Introduction}

IgE is the most significant component in the highly complex cellular and molecular events of immune response mediated by Th2 cells. In addition, besides playing a major role in immediate hypersensitivity there is evidence for a causal relationship between eosinophil recruitment and IgE [1]. IgE mediated allergic diseases have become public health concern as several organ systems of the body resulting in anaphylactic rhinoconjunctivitis, asthma, allergic gastroenteritis, allergic dermatitis, urticaria and even allergic shock [2]. More than $20 \%-50 \%$ people throughout the world have been affected by these diseases [3]. The measurement of serum total IgE (tIgE) concentration aids in the diagnostic assessment of patients with suspected allergic diseases [4]. However, racial and ethnic disparities have been reported in IIgE levels among different population around the globe such as in European, American, and some Asian populations. In addition, tIgE levels also differ depending on age and sex within the same racial and ethnic origin [5] [6] [7]. Therefore, tIgE levels which have been reported in previous studies in other countries may not be applicable to the Bangladeshi population in predicting whether an individual is having allergic disease or not.

Two previous studies from Bangladesh provided information regarding tIgE level but those were conducted among only asthma patients [8] [9]. However, distribution of tIgE levels among the general populations of Bangladesh who are clinically suspected cases of allergic diseases has not been reported to date. This study aims to quantitatively determine the serum tIgE levels in Bangladeshi population who were clinically suspected cases of allergic diseases and to find out the association of tIgE level with their age and gender.

\section{Methodology}

\subsection{Study Design}

This cross sectional descriptive exploratory study was conducted at Biomed Diagnostics Laboratory, Panthapath, Dhaka from March 2021-September 2021.

\subsection{Sampling Method and Sample Size}

Purposive sampling method was selected to collect a total of 402 blood samples.

\subsection{Study Population}

The study population was the patients referred to the Biomed Diagnostics Laboratory for diagnostic purpose to confirm an allergic trigger suspected on the basis of history and clinical evaluation irrespective of age and sex.

\subsection{Laboratory Methods}

Quantitative detection of total IgE antibodies (tIgE) in serum was done by Immunoblot assay manufactured by Proteome Tech Inc. (PROTIA ${ }^{\mathrm{TM}}$ Allergy-Q64S panel). PROTIA ${ }^{\mathrm{TM}}$ Allergy-Q64S panel is a diagnostic kit, based on the principle of enzyme immunoassay. 
Serum was separated by standard methods. Blood cells or any solid matters were removed by centrifugation. The serum samples were stored at $2^{\circ} \mathrm{C}-8^{\circ} \mathrm{C}$ when they were used for a short period (within 2 weeks) and at $-15^{\circ} \mathrm{C}$ for a longer duration.

All reagents were brought to room temperature around 30 minutes before use and mixed well. The other procedures were fully automated. The Q-STATION ELITE was set and it automatically performed the entire procedure of dispensing patient samples and reagents, incubation, washing, drying and measurement.

The amount of total IgE was expressed as IU/ml (in the range of $0-2000$ IU/ml) via Q-STATION ELITE.

\subsection{Statistical Analysis}

The data was imported into IBM SPSS Statistics 22 software (USA) for analysis.

\section{Result}

In the present study, the serum tIgE levels showed wide variations ranging from 5 to $2000 \mathrm{IU} / \mathrm{ml}$. Figure 1 shows distribution of total IgE (IU/ml) among 402 study subjects.

In the present study, the study population was stratified on the basis of their age and sex, and the total IgE levels in each of the age group in both sexes were studied. Figure 2 shows frequency distribution of the study population according to age (in years).

Furthermore, the mean tIgE level exhibited a declining tendency with an increase in age irrespective of gender. The highest mean tIgE levels being 721 $\mathrm{IU} / \mathrm{mL}$ was found in the $1-10$-year age group followed by the second and third decade of life whereas there was a progressive reduction in the mean level of tIgE, past the age of 40 . The lowest mean IIgE was observed among $70-80$ years age group. Figure 3 shows mean $\operatorname{tgE}(\mathrm{IU} / \mathrm{ml}$ ) according to 10 -year age group.

Among the 402 study subjects 206 (51\%) were female whereas 196 (49\%) were male. Male female ratio in the study population was 1:1.051. Depending on gender there was no difference in $\operatorname{tg} E$ levels.

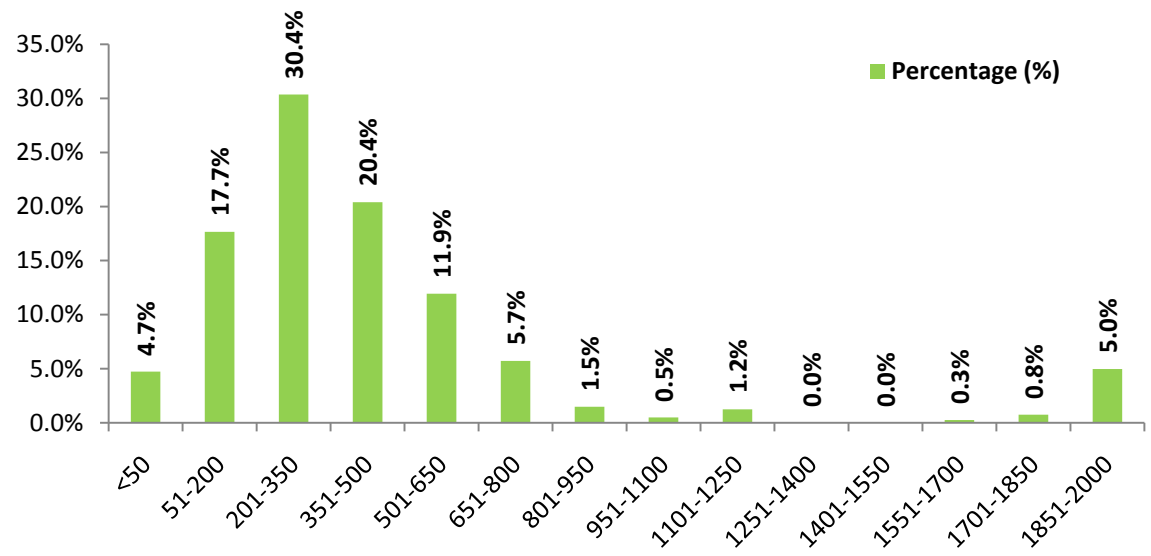

Figure 1. Distribution of total IgE (IU/ml) among 402 study subjects. 


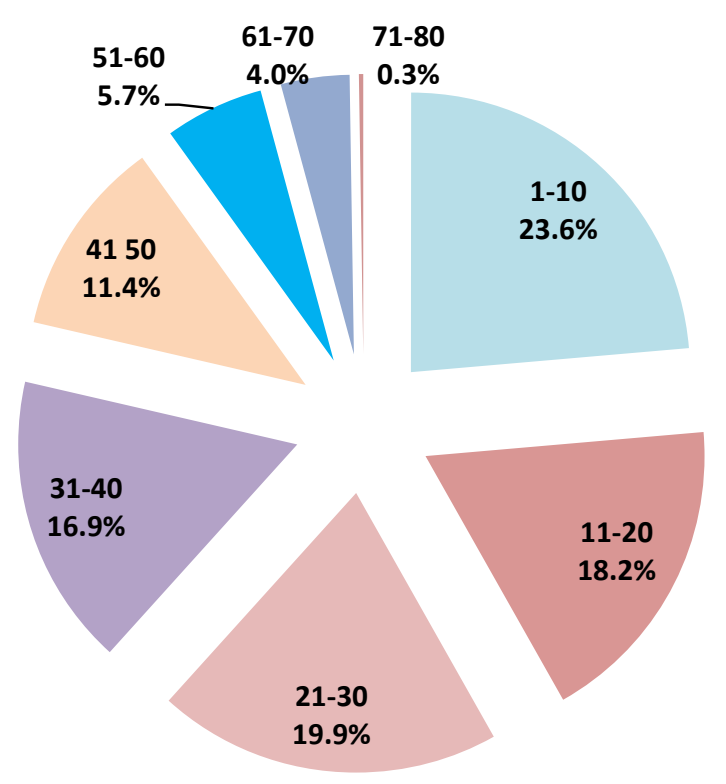

Figure 2. Age (years) distribution of study population.

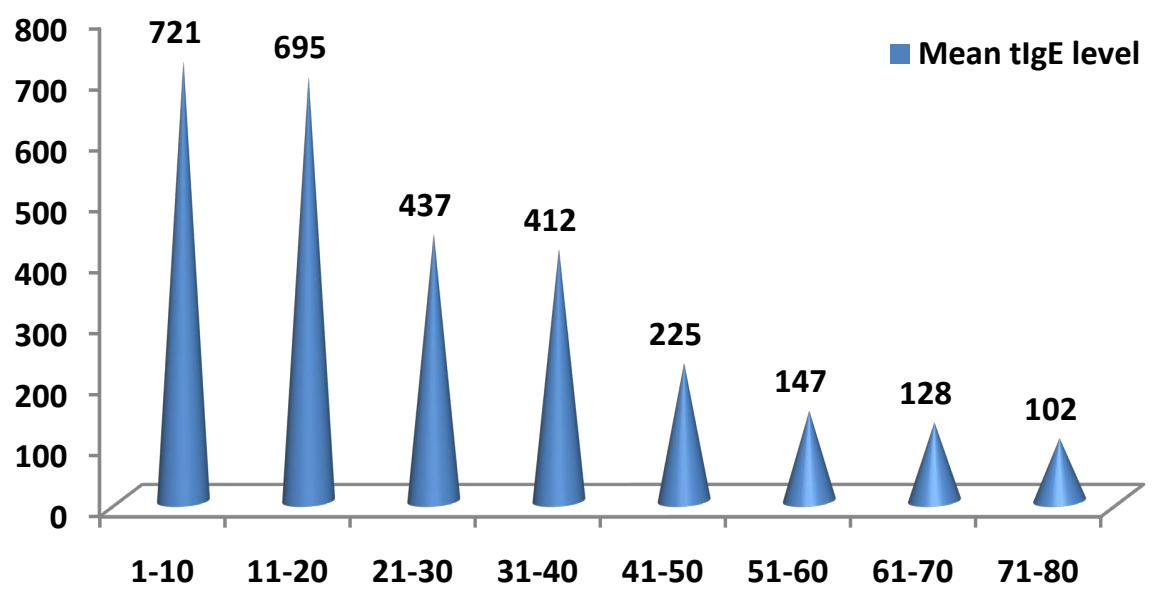

Figure 3. Mean total $\mathrm{IgE}(\mathrm{IU} / \mathrm{ml})$ according to 10 -year age group.

\section{Discussion}

In the present study, majority of the study population (30.35\%) showed tIgE levels within the range of $201-350 \mathrm{IU} / \mathrm{ml}$. Another study from Bangladesh reported that serum total IgE levels in adult patients with persistent allergic asthma were high with levels of $>150 \mathrm{IU} / \mathrm{mL}$ and a higher percentage of patients with total IgE $>400 \mathrm{IU} / \mathrm{mL}$ was observed in patients with severe asthma [7]. A study conducted in the department of Physiology, BSMMU, Dhaka and Asthma center, Mohakhali, Dhaka reported upper limits of normal value of total IgE as 100 $\mathrm{IU} / \mathrm{ml}$ and elevated total IgE was reported as $>100 \mathrm{Iu} / \mathrm{ml}$ [8]. In an Indian population, the total serum IgE levels showed wide variations ranging from 10 to 4000 $\mathrm{IU} / \mathrm{ml}$. The cause of this wide range could be due to the selection of 700 study subjects categorized in different groups such as 1) asthma with allergic rhinitis; 2) patients of asthma alone; 3) patients of allergic rhinitis; 4) first-degree rela- 
tives of patients, referred to as risk group; and 5) healthy volunteers (control group) taken together in the same study [10].

In 2015, Chang ML and colleagues assessed total IgE among 3721 Chinese population and significant reference range of serum total IgE was determined as $>100 \mathrm{IU} / \mathrm{ml}$ [11]. Ezeamuzie CI et al. conducted a study in Kuwait and concluded that, the reference range of total IgE values in normal adults ranged between $3.2-602.5 \mathrm{IU} / \mathrm{ml}[12]$. A study from UK revealed that, total IgE ranged from 0 to $80 \mathrm{kU} / \mathrm{litre}(\mathrm{IU} / \mathrm{ml}$ ) (which is, up to and within the UK protein reference laboratory's normal maximum for adults) [4]. Some other studies reported upper limits of normal levels of total IgE between 300 and $500 \mathrm{IU} / \mathrm{ml}$ [13]. As per our observations, the mean tIgE level exhibited a declining tendency with an increase in age. Some other studies corroborated the finding of an inverse relation between age and mean $\operatorname{tg} E$ level. Sharma $S$ et al reported that, in Indian population the highest mean IgE level being $642 \mathrm{IU} / \mathrm{mL}$ was reported in the 8 12-year age group whereas among the age group of 50 - 55 years the lowest mean IgE was found which was $352 \mathrm{IU} / \mathrm{ml}[10]$.

Among American population aged $38-82$ years, the observation of an age-associated decrease in the mean IgE levels was observed and the lowest mean tIgE was found in 70 - 80 years age [14]. Several other cross-sectional studies around the world revealed similar finding that, $\operatorname{tgE}$ was higher in younger subjects than older subjects reflecting a decline in tIgE sensitization with age [15]. Two reasons may explain this inverse relation between age and mean tIgE level: 1) There might be an association of age with a reduced ability to develop IgE-mediated allergic manifestations; 2) There might be reduced mast cell function. These factors strongly suggest a progressive natural desensitization to allergens in aged population [16] [17] [18]. In the present study, there was no association between tIgE and gender as the male female ratio in the study population was 1:1.051. However, effect of gender on tIgE levels is an area which remains largely unexplored to date. Some previous studies reported that generally males have higher tIgE levels than females and the reason of this observation could be the higher prevalence of smoking habit in men resulting in allergic respiratory diseases [19] [20] [21] [22]. On the contrary, Loza et al revealed that, gender has a profound effect on the regulation of Th2 cells, which may contribute to higher frequency of allergic diseases in females [23]. Another research showed that, independent of age and smoking habits, intake of oral contraceptive can increase the tIgE level, which indicates a relationship between hormone levels and allergic reactions [24].

Nevertheless, there were certain challenges and limitations of this study. Firstly, although increased tIgE level provides a general indication of allergic diseases, it could not be ignored that many clinically proven allergic individuals may have normal total IgE levels. Additionally, several possible confounding factors such as smoking status, parasite infestation, environmental exposures or seasonal variations can affect the tIgE level [25] [26]. However, in the current study we could not consider these factors. Studies on therapeutic anti-IgE anti- 
bodies (anti-IgE) are going on with a purpose of IgE neutralization in blood in addition to IgE $+\mathrm{B}$ cell reduction to combat type I hypersensitivity reactions. There has been one potential medicine namely Omalizumab which is FDA approved for treating patients with moderate-to-severe allergic asthma [27] [28]. Therefore, the pharmacological purposes of the anti-IgE therapy are certainly a propitious avenue for further research.

\section{Study Limitation}

The reason of mean tIgE level exhibiting a declining tendency with an increase in age could not be evaluated due to fund constraints.

\section{Conclusion}

To satisfy the need of properly screening allergic diseases and devising new therapeutics to prevent the major consequences of allergy the role of $\operatorname{tgE}$ is imperative. Although tIgE level among different populations around the world has been reported, it varies among different races and ethnicities [4] [24]. Our work strongly suggests that the allergic disease burden reduces with an increasing age whereas it does not vary depending on gender among Bangladeshi subjects.

\section{Recommendation}

Further researches with multivariable regression analysis in this topic are recommended.

\section{Acknowledgements}

We gratefully appreciate the help of laboratory personnel of the Biomed Diagnostics Laboratory Ltd.

\section{Conflicts of Interest}

The authors declare no conflicts of interest regarding the publication of this paper.

\section{References}

[1] Platts-Mills, T.A. (2001) The Role of Immunoglobulin E in Allergy and Asthma. American Journal of Respiratory and Critical Care Medicine, 164, S1-S5. https://doi.org/10.1164/ajrccm.164.supplement 1.2103024

[2] Fireman, P. (1995) Diagnosis of Allergic Disorders. Pediatrics in Review, 16, 178-183. https://doi.org/10.1542/pir.16-5-178

[3] Chowdhury, M.A., Kabir, A.L. and Alam, M.M. (2008) Allergic Rhinitis, Asthma and Atopic Diseases: Bangladesh Perspective. Bangladesh Journal of Otorhinolaryngology, 14, 66-70.

[4] Sinclair, D. and Peters, S. (2004) The Predictive Value of Total Serum IgE for a Positive Allergen Specific IgE Result. Journal of Clinical Pathology, 57, 956-959. https://doi.org/10.1136/jcp.2004.017681

[5] Litonjua, A.A., Celedón, J.C., Hausmann, J., Nikolov, M., Sredl, D., Ryan, L., 
Platts-Mills, T.A., Weiss, S.T. and Gold, D.R. (2005) Variation in Total and Specific IgE: Effects of Ethnicity and Socioeconomic Status. Journal of Allergy and Clinical Immunology, 115, 751-757. https://doi.org/10.1016/j.jaci.2004.12.1138

[6] Wegienka, G., Johnson, C.C., Zoratti, E. and Havstad, S. (2013) Racial Differences in Allergic Sensitization: Recent Findings and Future Directions. Current Allergy and Asthma Reports, 13, 255-261. https://doi.org/10.1007/s11882-013-0343-2

[7] Wohlford, E.M., Huang, P.F., Elhawary, J.R., Millette, L.A., Contreras, M.G., Witonsky, J., Holweg, C.T., Oh, S.S., Lee, C., Merenda, C. and Rabin, R.L. (2021) Racial/Ethnic Differences in Eligibility for Asthma Biologics among Pediatric Populations. Journal of Allergy and Clinical Immunology, 148, 1324-1331.

https://doi.org/10.1016/j.jaci.2021.09.005

[8] Muslemin, R., Khuda, K.M., Rahman, M.M., Islam, M.R. and Huq, S.R. (2019) Impact of Serum Total IgE and Disease Severity in Patients with Allergic Asthma: $\mathrm{NIDCH}$ in Dhaka. International Journal of Medical Research Professionals, 5, 152-155.

[9] Parveen, T., Begum, N. and Begum, S. (2009) Allergen Skin Test Reactivity and Serum Total IgE Level in Adult Bronchial Asthmatic Patients. Journal of Bangladesh Society of Physiologist, 4, 1-6.

[10] Sharma, S., Kathuria, P.C., Gupta, C.K., Nordling, K., Ghosh, B. and Singh, A.B. (2006) Total Serum Immunoglobulin E Levels in a Case-Control Study in Asthmatic/Allergic Patients, Their Family Members, and Healthy Subjects from India. Clinical \& Experimental Allergy, 36, 1019-1027. https://doi.org/10.1111/j.1365-2222.2006.02525.x

[11] Chang, M.L., Cui, C., Liu, Y.H., Pei, L.C. and Shao, B. (2015) Analysis of Total Immunoglobulin E and Specific Immunoglobulin E of 3,721 Patients with Allergic Disease. Biomedical Reports, 3, 573-577. https://doi.org/10.3892/br.2015.455

[12] Ezeamuzie, C.I., Al-Ali, S.F., Al-Dowaisan, A., Khan, M., Hijazi, Z. and Thomson, M.S. (1999) Reference Values of Total Serum IgE and Their Significance in the Diagnosis of Allergy among the Young Adult Kuwaiti Population. Clinical and Experimental Allergy, 29, 375-381. https://doi.org/10.1046/j.1365-2222.1999.00463.x

[13] Grigoreas, C., Pappas, D., Galatas, I.D., Kollias, G., Papadimos, S. and Papadakis, P. (1993) Serum Total IgE Levels in a Representative Sample of a Greek Population: I. Correlation with Age, Sex, and Skin Reactivity to Common Aeroallergens. Allergy, 48, 142-146. https://doi.org/10.1111/j.1398-9995.1993.tb00703.x

[14] Criqui, M.H., Seibles, J., Hamburger, R.N., Coughlin, S.S. and Gabriel, S. (1990) Epidemiology of Immunoglobulin E Levels in a Defined Population. Annals of Allergy, 64, 308-313.

[15] Jarvis, D., Luczynska, C., Chinn, S., Potts, J., Sunyer, J., Janson, C., Svanes, C., Künzli, N., Leynaert, B., Heinrich, J. and Kerkhof, M. (2005) Change in Prevalence of IgE Sensitization and Mean Total IgE with Age and Cohort. Journal of Allergy and Clinical Immunology, 116, 675-682. https://doi.org/10.1016/j.jaci.2005.05.009

[16] Orren, A. and Dowdle, E.B. (1975) The Effects of Sex and Age on Serum IgE Concentrations in Three Ethnic Groups. International Archives of Allergy and Immunology, 48, 824-835. https://doi.org/10.1159/000231370

[17] Hanneuse, Y., Delespesse, G., Hudson, D., De Halleux, F. and Jacques, J.M. (1978) Influence of Ageing on IgE-Mediated Reactions in Allergic Patients. Clinical \& EXperimental Allergy, 8, 165-174. https://doi.org/10.1111/j.1365-2222.1978.tb00461.x

[18] Pupil, P. and Berna, M. (1976) Skin Tests for 48/80 and Histamine. Annales de I Anesthesiologie Francaise, 17, 199-208. 
[19] Omenaas, E., Bakke, P., Elsayed, S., Hanoa, R. and Gulsvik, A. (1994) Total and Specific Serum IgE Levels in Adults: Relationship to Sex, Age and Environmental Factors. Clinical \& Experimental Allergy, 24, 530-539. https://doi.org/10.1111/j.1365-2222.1994.tb00950.x

[20] Kerkhof, M., Droste, J.H., de Monchy, J.G., Schouten, J.P. and Rijcken, B. (1996) Distribution of Total Serum IgE and Specific IgE to Common Aeroallergens by Sex and Age, and Their Relationship to Each Other in a Random Sample of the Dutch General Population Aged 20-70 Years. Dutch ECRHS Group, European Community Respiratory Health Study. Allergy, 51, 770-776. https://doi.org/10.1111/j.1398-9995.1996.tb00021.x

[21] Wuthrich, B., Schindler, C., Medici, T.C., Zellweger, J.P. and Leuenberger, P. (1996) IgE Levels, Atopy Markers and Hay Fever in Relation to Age, Sex and Smoking Status in a Normal Adult Swiss Population. SAPALDIA (Swiss Study on Air Pollution and Lung Diseases in Adults) Team. International Archives of Allergy and Immunology, 111, 396-402. https://doi.org/10.1159/000237398

[22] Barbee, R.A., Halonen, M., Lebowitz, M. and Burrows, B. (1981) Distribution of IgE in a Community Population Sample: Correlations with Age, Sex, and Allergen Skin Test Reactivity. Journal of Allergy and Clinical Immunology, 68, 106-111. https://doi.org/10.1016/0091-6749(81)90167-6

[23] Loza, M.J., Foster, S., Bleecker, E.R., Peters, S.P., Penn, R.B. (2010) Asthma and Gender Impact Accumulation of T Cell Subtypes. Respiratory Research, 11, Article No. 103. https://doi.org/10.1186/1465-9921-11-103

[24] Jensen-Jarolim, E. and Untersmayr, E. (2008) Gender-Medicine Aspects in Allergology. Allergy, 63, 610-615. https://doi.org/10.1111/j.1398-9995.2008.01645.x

[25] Medeiros, D., Silva, A.R., Rizzo, J.A., Motta, M.E. and Sarinho, E. (2006) Total IgE Level Is Associated with Anti-Ascaris Specific IgE but Not with Stool Parasites or Blood Eosinophilia in Young Allergic Patients in a Tropical Region (Recife-Brazil). Journal of Allergy and Clinical Immunology, 117, S200. https://doi.org/10.1016/j.jaci.2005.12.788

[26] Wang, J., Wu, J.S. and He, L. (2015) Allergic Disease Epidemiology. In: Tao, A. and Raz, E., Eds., Allergy Bioinformatics, Springer, Dordrecht, 15-41. https://doi.org/10.1007/978-94-017-7444-4 2

[27] Chang, T.W., Wu, P.C., Hsu, C.L. and Hung, A.F. (2007) Anti-IgE Antibodies for the Treatment of IgE-Mediated Allergic Diseases. Advances in Immunology, 93, 63-119.

[28] Hu, J., Chen, J., Ye, L., Cai, Z., Sun, J. and Ji, K. (2018) Anti-IgE Therapy for IgE-Mediated Allergic Diseases: From Neutralizing IgE Antibodies to Eliminating $\mathrm{IgE}^{+}$B Cells. Clinical and Translational Allergy, 8, Article No. 27. https://doi.org/10.1186/s13601-018-0213-Z 